\title{
CENTRAL LIMIT THEOREM FOR HITTING TIMES OF FUNCTIONALS OF MARKOV JUMP PROCESSES
}

\author{
Christian Paroissin ${ }^{1}$ And Bernard YCART $^{1}$
}

\begin{abstract}
A sample of i.i.d. continuous time Markov chains being defined, the sum over each component of a real function of the state is considered. For this functional, a central limit theorem for the first hitting time of a prescribed level is proved. The result extends the classical central limit theorem for order statistics. Various reliability models are presented as examples of applications.
\end{abstract}

Mathematics Subject Classification. 60F05, 60J25, 60K10.

Received July 18, 2003. Revised May 24, 2003 and October 13, 2003.

\section{INTRODUCTION}

Models made of $n$-tuples of i.i.d. Markov chains appear in different domains of applications. They can represent locations of a set of customers in a closed Jackson network [21], the current state of a parallel MCMC algorithm [6], or a description of a coherent system in reliability [3]. We shall use here the reliability interpretation as a support for intuition, although the applicability of our results is not restricted to that area. In [19], the relaxation time of samples of i.i.d. Markov chains was studied and a cutoff phenomenon was shown to occur. In [20] the hitting time of functionals of such a sample was used for detecting access to equilibrium, thus providing stopping rules for MCMC algorithms. Our main focus here is on the behavior of those functionals far from equilibrium.

Let $X=\{X(t), t \geq 0\}$ be a Markov chain in continuous time, with values in a finite state space $E$. The random variable $X(t)$ will be interpreted as the state at time $t$ of a component bound to degradations and failures. Consider a system made of $n$ independent such components. Its state will be described by a $n$-tuple $\tilde{X}=\left(X_{1}, \ldots, X_{n}\right)$ where the $X_{i}$ 's are independent copies of $X$. This process is again a continuous time Markov chain $\{\tilde{X}(t), t \geq 0\}$, with values in the product space $E^{n}$. Let $f$ be a function from $E$ to $\mathbb{R}$. In the reliability interpretation, $f(e)$ measures a level of degradation for a component in state $e$. The total degradation of the system in state $\eta=\left(\eta_{i}\right) \in E^{n}$ will be measured by the sum $\sum_{i} f\left(\eta_{i}\right)$. So we shall focus on the process $S_{n}=\left\{S_{n}(t), t \geq 0\right\}$, where $S_{n}(t)$ is the total degradation of the system at time $t$ :

$$
S_{n}(t)=\sum_{i=1}^{n} f\left(X_{i}(t)\right)
$$

Keywords and phrases. Central limit theorem, hitting time, reliability, failure time.

${ }^{1}$ Laboratoire MAP5, 45 rue des Saints-Pères, 75270 Paris Cedex 06, France;

e-mail: parc@math-info.univ-paris5.fr and ycart@math-info.univ-paris5.fr 
It is natural to consider the instants at which $S_{n}(t)$ reaches a prescribed level of degradation. Let $k=(k(n))$ be a sequence of reals. Our main object is the failure time $T_{n}$, defined as:

$$
T_{n}=\inf \left\{t \geq 0 ; S_{n}(t) \geq k(n)\right\} .
$$

In the particular case where $E=\{$ working, failed $\}$ (binary components), and $f$ is the indicator of a failed component, then $S_{n}(t)$ simply counts the number of failed components at time $t$, and our system is a so-called " $k$-out-of- $n$ " system [4].

Let $e_{0}=X(0)$ be the initial state of all components (in reliability $e_{0}$ will be the perfect state). We shall denote by $m(t)$ (respectively: $v(t)$ ) the expectation (resp.: the variance) of the degradation at time $t$.

$$
m(t)=\mathbb{E}\left[f(X(t)) \mid X(0)=e_{0}\right], v(t)=\operatorname{Var}\left[f(X(t)) \mid X(0)=e_{0}\right] .
$$

Since all components are i.i.d., the average degradation $S_{n}(t) / n$ converges in probability to its expectation $m(t)$. We shall assume that $m(t)$ is strictly increasing on the interval $[0, \tau]$, with $0<\tau \leq+\infty$ (the degradation starting from the perfect state increases on average). We consider a "mean degradation level" $\alpha$, such that $m(0)<\alpha<m(\tau)$. Assume the threshold $k(n)$ is such that:

$$
k(n)=\alpha n+o(\sqrt{n}) .
$$

The failure time $T_{n}$ has to be close to the instant at which $m(t)$ crosses level $\alpha$ :

$$
t_{\alpha}=\inf \{t ; m(t)=\alpha\}
$$

Our main result is the following central limit theorem.

Theorem 1.1. Under the above hypotheses,

$$
\sqrt{n}\left(T_{n}-t_{\alpha}\right) \underset{n \longrightarrow+\infty}{\stackrel{\mathcal{L}}{\longrightarrow}} \mathcal{N}\left(0, \sigma_{\alpha}^{2}\right),
$$

with:

$$
\sigma_{\alpha}^{2}=\frac{v\left(t_{\alpha}\right)}{\left(m^{\prime}\left(t_{\alpha}\right)\right)^{2}} .
$$

Section 2 is devoted to the proof of Theorem 1.1. It starts with a functional central limit theorem for $S_{n}$ (Prop. 2.1), which is an application of more general results by Hahn [10] and Whitt [18], and closely related to the huge corpus of diffusion approximations (see Ethier and Kurtz [9] for a general reference). Then the Skorohod-Dudley-Wichura representation theorem (Dudley [8] or Pollard [15]) is used both to prove that the distributions of $\sqrt{n}\left(T_{n}-t_{\alpha}\right)$ are a tight sequence and to characterize the limit.

In Section 3 we give some examples of reliability models to which our results apply. The simplest of them is the $k$-out-of- $n$ model, with binary components having exponential failure and repair times (see Bhat [5]). Our results also apply when the failure and repair times have phase type distributions $[12,13,17]$, and we shall study the case where a deterministic repair time is approximated by an Erlang distribution. In the particular case of a $k$-out-of- $n$ system with non-repairable components, the failure time $T_{n}$ of the system is an order statistic of failure times for the components. The central limit theorem for order statistics is well known (see for instance [16]). Here it comes out as a particular case of Theorem 1.1. A model with 3 state components studied by Pham et al. [14], will be considered next.

\section{Asymptotic Results}

We shall first precise some notations, then state the central limit theorem for the degradation process $S_{n}$ (Prop. 2.1). Next, the proof of Theorem 1.1 will be completed. 
As explained before, the state of one component is described by a continuous time Markov chain $X=$ $\{X(t), t \geq 0\}$. Its infinitesimal generator will be denoted by $\Lambda$, and its transition rate from state $e$ to state $e^{\prime}$ by $\lambda_{e e^{\prime}}$. It is well known (see for instance Bhat [5], Sect. 7.7, pp. 219-224), that the distribution at time $t$ of $X(t)$, starting from $e_{0}$, is the $e_{0}$-th column of the matrix $\exp \left({ }^{t} \Lambda t\right)$. It will be denoted by $p(t)=\left(p_{e}(t)\right), e \in E$. (All our vectors are columns and ${ }^{t} A$ denotes matrix transpose.)

We consider a $n$-tuple $\tilde{X}=\left(X_{1}, \ldots, X_{n}\right)$ of independent copies of $X$. It is a continuous time Markov chain on $E^{n}$, and its infinitesimal generator is the Kronecker sum of $n$ copies of $\Lambda$ ([19], pp. 92-94). We shall also need the "counting process" $N_{n}$, defined as the number of components of $\tilde{X}$ in each state of $E: N_{n}=\left\{N_{n}(t), t \geq 0\right\}$, with:

$$
N_{n}(t)=\left(N_{n, e}(t)\right), e \in E \text { and } N_{n, e}(t)=\sum_{i=1}^{n} \mathbb{1}_{e}\left(X_{i}(t)\right),
$$

where $\mathbb{1}_{e}$ denotes the indicator function of state $e$. Thus $N$ appears as the sum of $n$ independent copies of the Markov chain $\mathbb{1}(X)=\left(\mathbb{1}_{e}(X)\right), e \in E$. The expectation of $\mathbb{1}(X(t))$ is $p(t)$. For $0 \leq s \leq t$ the covariance matrix of $\mathbb{1}(X(s))$ with $\mathbb{1}(X(t))$, denoted by $K(s, t)$, can be expressed as:

$$
K(s, t)=\operatorname{diag}(p(s)) \exp (\Lambda(t-s))-p(s)^{t} p(t) .
$$

The degradation function $f$, from $E$ to $\mathbb{R}$ will be identified to the vector $(f(e)), e \in E$. The degradation at time $t$ of one component can be written as a linear combination of $\mathbb{1}(X(t))$ :

$$
f(X(t))={ }^{t} f \mathbb{1}(X(t)) .
$$

Thus its expectation at time $t$ is:

$$
m(t)={ }^{t} f p(t)
$$

For $0 \leq s \leq t$ the covariance function is:

$$
\operatorname{Cov}[f(X(s)), f(X(t))]={ }^{t} f K(s, t) f .
$$

In particular for $s=t$, the variance of the degradation at time $t$ is:

$$
v(t)=\operatorname{Var}\left[f(X(t)) \mid X(0)=e_{0}\right]={ }^{t} f K(t, t) f .
$$

It is easy to prove that the counting process $N_{n}$ is again a continuous time Markov chain (see [21] p. 296 for the Jackson network interpretation of $N_{n}$ ). Since the $X_{i}$ 's are independent, the distribution of the random vector $N_{n}(t)$ is multinomial with parameters $n$ and $p(t)$. Of course, the "total degradation" process $S_{n}(t)$ is a linear combination of $N_{n}(t): S_{n}(t)={ }^{t} f N_{n}(t)$.

As sums of independent processes, $S_{n}$ as well as $N_{n}$ both satisfy functional central limit theorems. We shall only need it for $S_{n}$.

Proposition 2.1. For all $n$, let $Z_{n}=\left\{Z_{n}(t), t \geq 0\right\}$ be defined by:

$$
Z_{n}(t)=\frac{1}{\sqrt{n}}\left(S_{n}(t)-n m(t)\right)
$$

The sequence of processes $\left(Z_{n}\right)_{n \in \mathbb{N}}$ converges in distribution to a centered Gaussian process $Z$ with covariance function defined for $0 \leq s \leq t$ by:

$$
\operatorname{Cov}[Z(s), Z(t)]={ }^{t} f K(s, t) f,
$$

where the covariance matrix $K(s, t)$ is given by (2.1). Moreover, $Z$ has continuous sample paths. 
This CLT is a straightforward application of a result proved by Hahn (Cor. 3, p. 95 in [10]), using Whitt's tools (Sect. 1, p. 68 and Sect. 4, p. 78 in [18]).

Proposition 2.1 shows that the degradation process $S$ should remain at distance $O(\sqrt{n})$ from the deterministic function $n m$. Our assumption is that $m$ is strictly increasing over the interval $[0, \tau]$, where $0<\tau \leq+\infty$. For $m(0)<\alpha<m(\tau)$, there exists a unique instant $t_{\alpha} \in[0, \tau]$ such that $m\left(t_{\alpha}\right)=\alpha$. We want to study the hitting time of level $k=k(n)$, where:

$$
k(n)=\alpha n+o(\sqrt{n}) .
$$

It is defined as:

$$
T_{n}=\inf \left\{t \geq 0 ; S_{n}(t) \geq k(n)\right\}
$$

It is intuitively clear that $T_{n}$ should be close to $t_{\alpha}$, with an error of order $1 / \sqrt{n}$.

Let us now complete the proof of Theorem 1.1.

Proof. The convergence in distribution of $Z_{n}$ does not directly imply the CLT for $T_{n}$. But the SkorohodDudley-Wichura representation theorem is a much stronger result (see Th. 11.7.2 of Dudley [8], or Pollard [15], Sect. IV.3). It implies that there exist versions $S_{n}^{*}$ of $S_{n}$ and $Z^{*}$ of $Z$, and non-decreasing functions $\phi_{n}$ such that for any fixed $s$ :

and

$$
\lim _{n \rightarrow \infty} \sup _{0 \leq t \leq s}\left|\frac{S_{n}^{*}(t)-n m(t)}{\sqrt{n}}-Z^{*}\left(\phi_{n}(t)\right)\right|=0 \quad \text { a.s. }
$$

$$
\lim _{n \rightarrow \infty} \sup _{0 \leq t \leq s}\left|\phi_{n}(t)-t\right|=0 \quad \text { a.s. }
$$

Since $Z^{*}$ has continuous paths, it is uniformly continuous on $[0, s]$, and hence:

$$
\lim _{n \rightarrow \infty} \sup _{0 \leq t \leq s}\left|Z_{n}^{*}(t)-Z^{*}(t)\right|=0 \quad \text { a.s. }
$$

with

In other words, we have

$$
Z_{n}^{*}(t)=\frac{S_{n}^{*}(t)-n m(t)}{\sqrt{n}}
$$

uniformly over $[0, s]$ for any $s$.

$$
Z_{n}^{*}(t)=Z^{*}(t)+o(1) \quad \text { a.s. }
$$

We shall first use (2.3) to prove that the distributions of $\sqrt{n}\left(T_{n}-t_{\alpha}\right)$ are a tight sequence. Let $c$ be a positive constant. On the one hand, if $S_{n}\left(t_{\alpha}+c / \sqrt{n}\right) \geq k(n)$, then $T_{n} \leq t_{\alpha}+c / \sqrt{n}$. Thus:

$$
\begin{aligned}
\mathbb{P}\left[\sqrt{n}\left(T_{n}-t_{\alpha}\right) \leq c\right] & \geq \mathbb{P}\left[S_{n}^{*}\left(t_{\alpha}+c / \sqrt{n}\right) \geq k(n)\right] \\
& =\mathbb{P}\left[Z_{n}^{*}\left(t_{\alpha}+c / \sqrt{n}\right) \geq \sqrt{n}\left(\alpha-m\left(t_{\alpha}+c / \sqrt{n}\right)\right)+o(1)\right] \\
& =\mathbb{P}\left[Z_{n}^{*}\left(t_{\alpha}+c / \sqrt{n}\right) \geq-c m^{\prime}\left(t_{\alpha}\right)+o(1)\right] \\
& =\mathbb{P}\left[Z^{*}\left(t_{\alpha}\right) \geq-c m^{\prime}\left(t_{\alpha}\right)\right]+o(1),
\end{aligned}
$$

using (2.3) and the continuity of $Z^{*}$. Since $m^{\prime}\left(t_{\alpha}\right)>0$, we obtain that:

$$
\lim _{c \rightarrow \infty} \liminf _{n \rightarrow \infty} \mathbb{P}\left[\sqrt{n}\left(T_{n}-t_{\alpha}\right) \leq c\right]=1
$$

On the other hand, we have:

$$
\mathbb{P}\left[\sqrt{n}\left(T_{n}-t_{\alpha}\right) \leq-c\right]=\mathbb{P}\left[\exists t \leq t_{\alpha}-c / \sqrt{n}, Z_{n}^{*}(t) \geq \sqrt{n}(\alpha-m(t))+o(1)\right] .
$$


But since the function $m$ is increasing, for all $t \leq t_{\alpha}-c / \sqrt{n}$ we have:

$$
\sqrt{n}(\alpha-m(t)) \geq \sqrt{n}\left(\alpha-m\left(t_{\alpha}-c / \sqrt{n}\right)\right)=c m^{\prime}\left(t_{\alpha}\right)+o(1) .
$$

Hence:

$$
\begin{aligned}
\mathbb{P}\left[\sqrt{n}\left(T_{n}-t_{\alpha}\right) \leq-c\right] & \leq \mathbb{P}\left[\exists t \leq t_{\alpha}-c / \sqrt{n}, Z_{n}^{*}(t) \geq c m^{\prime}\left(t_{\alpha}\right)+o(1)\right] \\
& \leq \mathbb{P}\left[\exists t \leq t_{\alpha}, Z_{n}^{*}(t) \geq c m^{\prime}\left(t_{\alpha}\right)+o(1)\right] \\
& =\mathbb{P}\left[\exists t \leq t_{\alpha}, Z^{*}(t) \geq c m^{\prime}\left(t_{\alpha}\right)+o(1)\right]+o(1) .
\end{aligned}
$$

The process $Z^{*}$ being a.s. bounded on any compact set and $m^{\prime}(t)$ being positive on $[0, \tau]$, we deduce that:

$$
\lim _{c \rightarrow \infty} \limsup _{n \rightarrow \infty} \mathbb{P}\left[\sqrt{n}\left(T_{n}-t_{\alpha}\right) \leq-c\right]=0 .
$$

Now (2.4) and (2.5) mean that the sequence of distributions of $\left(\sqrt{n}\left(T_{n}-t_{\alpha}\right)\right)$ is tight. Hence to conclude it is enough to check the limit.

Using again (2.3), together with the almost sure continuity of $Z^{*}$ yields:

$$
\begin{aligned}
S_{n}^{*}\left(t_{\alpha}+u / \sqrt{n}\right) & =n m\left(t_{\alpha}+u / \sqrt{n}\right)+\sqrt{n} Z^{*}\left(t_{\alpha}+u / \sqrt{n}\right)+o(\sqrt{n}) \quad \text { a.s. } \\
& =n \alpha+u \sqrt{n} m^{\prime}\left(t_{\alpha}\right)+\sqrt{n} Z^{*}\left(t_{\alpha}\right)+o(\sqrt{n}) \quad \text { a.s. }
\end{aligned}
$$

Therefore:

$$
\begin{aligned}
\inf \left\{u ; S_{n}^{*}\left(t_{\alpha}+u / \sqrt{n}\right) \geq k(n)\right\} & =\inf \left\{u ; u \sqrt{n} m^{\prime}\left(t_{\alpha}\right)+\sqrt{n} Z^{*}\left(t_{\alpha}\right)+o(\sqrt{n}) \geq 0\right\} \\
& =-\frac{Z^{*}\left(t_{\alpha}\right)}{m^{\prime}\left(t_{\alpha}\right)}+o(1) .
\end{aligned}
$$

The distribution of $-Z^{*}\left(t_{\alpha}\right) / m^{\prime}\left(t_{\alpha}\right)$ is normal with mean 0 and variance $\sigma_{\alpha}^{2}$, hence the result.

This proof was actually given by the referee. In the preliminary version of this article the proof of Theorem 1.1 was based on an exponential inequality for the left tail of $T_{n}$. It was much longer and less elegant.

\section{Reliability MODELS}

As explained in [14], the notion of redundancy is central to many problems in reliability. This justifies the introduction of models made of a large number of identical components. The most classical case is that of binary components: each one can be either working or failed, and the function $f$ is 0 if the component is working, and 1 otherwise. All components are supposed to be working at time $t=0$. The total degradation of the system is simply the number of failed components, and the breakdown occurs when the number of failed components passes a certain threshold $k$. These systems are called " $k$-out-of- $n$ " ( $c f$. for instance Barlow and Proschan [4] or Cocozza-Thivent [7]). The application of our results to $k$-out-of- $n$ systems of binary components is presented in the first subsection. The second one will study the generalization to multi-state components.

\subsection{Binary components}

When $f(E)=\{0,1\}$, the degradation at time $t$ is a Bernoulli random variable. Its expectation $m(t)$ is the probability for one component to be failed at time $t$, and its variance $v(t)$ is $m(t)(1-m(t))$. Recall that the 
instant at which the mean degradation $m$ first passes level $\alpha$ is denoted by $t_{\alpha}$. The threshold $k(n)$ is taken to be $\alpha n+o(\sqrt{n})$. Theorem 1.1 states that the asymptotic distribution of $\sqrt{n}\left(T_{n}-t_{\alpha}\right)$ is Gaussian, with variance:

$$
\sigma_{\alpha}^{2}=\frac{v\left(t_{\alpha}\right)}{m^{\prime}\left(t_{\alpha}\right)^{2}}
$$

In the particular case of binary components, $v\left(t_{\alpha}\right)=\alpha(1-\alpha)$. So the knowledge of $m(t)$ suffices to determine the asymptotic distribution of the failure time $T_{n}$. In reliability theory $m$ is the "non-availability" of one component.

The simplest expressions are obtained when the failure and repair times of each component are exponentially distributed, with respective rates $\lambda$ and $\mu$. Easy computations (see for instance [5], Sect. 7.5, pp. 210-226 and Sect. 15.6, p. 471) lead to:

$$
m(t)=\frac{\lambda}{\lambda+\mu}-\frac{\lambda}{\lambda+\mu} \mathrm{e}^{-(\lambda+\mu) t}
$$

This function is strictly increasing over $[0,+\infty]$. Thus one can choose $\tau=+\infty$, and Theorem 1.1 can be applied for any $\alpha$ such that $0<\alpha<\frac{\lambda}{\lambda+\mu}$. We get:

$$
t_{\alpha}=-\frac{1}{\lambda+\mu} \log \left(1-\frac{\alpha(\lambda+\mu)}{\lambda}\right)
$$

Thus the asymptotic variance $\sigma_{\alpha}^{2}$ is:

$$
\sigma_{\alpha}^{2}=\frac{\alpha(1-\alpha)}{(\lambda-\alpha(\lambda+\mu))^{2}} .
$$

Consider now binary components having non-exponential repair or failure times. Since they are not Markovian, our results do not apply directly. However, any distribution can be approximated by a phase-type distribution. There exists an extensive literature on this subject, for which important references are Neuts' [12, 13] and Tijms' [17] books. These techniques coming from queuing theory are often called matrix-analytic methods (see for instance Asmussen's book [1] and his recent survey on the subject [2]). Phase-type distributions are laws of sojourn times in continuous time Markov chains. Their set is dense in the set of distributions with values on $\mathbb{R}^{+}([12]$, p. 243).

Assume one has to study binary components for which the failure and repair times have phase-type distributions. For one component, one can replace each one of the two states by a set of "fictitious" states, designed so as to make the sojourn time in all fictitious working (resp.: failed) states distributed as the prescribed failure (resp.: repair) time. Of course the value of the degradation function $f$ will be 0 (resp.: 1 ) on all fictitious working (resp.: failed) states.

Replacing the initial distributions by phase-type approximations before applying Theorem 1.1, supposes that the law of the failure time $T_{n}$ should not change by much. We shall not formalize that intuition. We shall only show that it can be supported by simulation experiments on a particular case.

Suppose that the repair time of a component is deterministic, equal to $1 / \mu$, whereas its failure time is exponential with parameter $\lambda$ as before. The behavior of such a binary component is a so called "alternate renewal process". It can be checked (see e.g. [11], pp. 311-313) that the probability of failure at time $t$ has the following expression:

$$
m(t)=1-\sum_{j=0}^{+\infty} \frac{\lambda^{j}}{j !}\left(t-\frac{j}{\mu}\right)^{j} \mathrm{e}^{-\lambda\left(t-\frac{j}{\mu}\right)} \mathbb{1}_{\left[\frac{j}{\mu},+\infty\right]}(t) .
$$

Figure 1 represents $m(t)$ together with one trajectory of the process $S_{n} / n$, for a system with $n=1000$ components, the failure and repair rates being $\lambda=1$ and $\mu=1$. Notice that $m$ is strictly increasing over the interval $[0 ; \mu]$, then decreases and oscillates towards its asymptotic value $\frac{\lambda}{\lambda+\mu}$. 


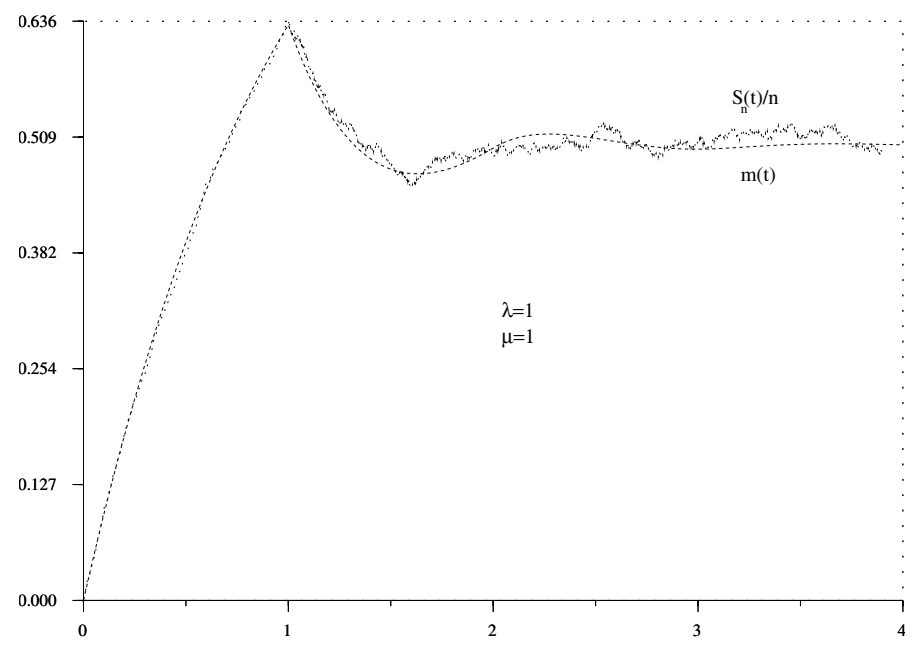

FIGURE 1. Non-availability and simulation of a system with deterministic repair times.

TABLE 1. Maximal difference between the exact value of $m$ and a phase-type approximation, for a binary system with deterministic repair times.

\begin{tabular}{|c||c|c|c|c|c|c|c|c|c|c|}
\hline$r$ & 10 & 20 & 30 & 40 & 50 & 60 & 70 & 80 & 90 & 100 \\
\hline \hline Max. diff. & 0.091 & 0.069 & 0.059 & 0.053 & 0.048 & 0.044 & 0.041 & 0.039 & 0.037 & 0.035 \\
\hline
\end{tabular}

To approximate the Dirac mass at $1 / \mu$ by a phase-type distribution, it suffices to consider an Erlang distribution with parameters $r$ and $r \mu$, for $r$ large enough. So we will consider a Markov chain $X$ on the state space $E=\{0, \ldots, r\}$, having the following infinitesimal generator:

$$
\Lambda_{r}=\left(\begin{array}{ccccc}
-\lambda & \lambda & 0 & \ldots & 0 \\
0 & -r \mu & r \mu & \ddots & \vdots \\
\vdots & \ddots & \ddots & \ddots & 0 \\
0 & & \ddots & -r \mu & r \mu \\
r \mu & 0 & \ldots & 0 & -r \mu
\end{array}\right)
$$

State 0 is the perfect state and states $1, \ldots, r$ represent fictitious failed states. The Laplace transform of the marginal distribution $p(t)$ is easily deduced from the Chapmann-Kolmogorov system. Denoting by $\mathscr{L}_{m}$ the Laplace transform of the mean degradation $m$, one gets:

$$
\mathscr{L}_{m}(s)=\frac{1}{s}-\frac{1}{s+\lambda-\lambda\left(\frac{r \mu}{s+r \mu}\right)^{r}} .
$$

The Laplace transform of $m^{\prime}$ is immediately deduced from $\mathscr{L}_{m}$. However $m$ and $m^{\prime}$ do not have tractable explicit expressions, and must be computed numerically.

As an example, we chose again $\lambda=\mu=1$. For $r=10,20, \ldots, 100$, we computed the maximal difference between the exact value of $m$ (formula (3.1)), and its phase-type approximation, by numerically solving the Chapmann-Kolmogorov system. The results are given in Table 1. 
TABLE 2. Comparison of empirical distributions of hitting times, with asymptotic distributions on a phase type approximation.

\begin{tabular}{|c||c|c|c|c|}
\hline$\alpha$ & $t_{\alpha}$ & $\sigma_{\alpha}^{2}$ & K.-S. stat. & $p$-value \\
\hline \hline 0.05 & 0.051 & 0.053 & 0.920 & 0.366 \\
\hline 0.10 & 0.105 & 0.111 & 1.004 & 0.266 \\
\hline 0.15 & 0.163 & 0.176 & 0.829 & 0.497 \\
\hline 0.20 & 0.223 & 0.25 & 0.827 & 0.501 \\
\hline 0.25 & 0.288 & 0.333 & 0.773 & 0.589 \\
\hline 0.30 & 0.357 & 0.428 & 0.792 & 0.557 \\
\hline 0.35 & 0.431 & 0.538 & 1.003 & 0.267 \\
\hline 0.40 & 0.511 & 0.666 & 0.681 & 0.743 \\
\hline 0.45 & 0.598 & 0.818 & 0.957 & 0.318 \\
\hline 0.50 & 0.693 & 1.000 & 0.703 & 0.706 \\
\hline
\end{tabular}

However, the maximal difference corresponds to the sharp peak of $m(t)$ and the approximation is notably better for smaller values of $t$. From then on, we fixed $r=100$ and $n=500$, and considered the levels $k(n)=\lfloor\alpha n\rfloor$, for $\alpha=0.05,0.1, \ldots, 0.5$. For those $\alpha$ 's, the exact values of $t_{\alpha}$ agreed with those of the phase-type approximation up to the fourth decimal. For each value of $\alpha$, the mean hitting time $t_{\alpha}$ and the variance $\sigma_{\alpha}^{2}$ were numerically computed for the phase-type approximation. Then we simulated 100 independent trajectories of the system with deterministic repair times, and recorded for each one the hitting time $T_{n}$ of the different levels $k(n)$. For each value of $\alpha$, the empirical distribution of $T_{n}$ was compared with the asymptotic normal distribution given in Theorem 1.1, by computing the $p$-value of the Kolmogorov-Smirnov test. The results are given in Table 2. With any usual risk, the goodness-of-fit hypothesis is accepted in all cases. Notice that since hitting times were computed along the same trajectories, their values for different $\alpha$ 's are not independent.

Let us now consider binary, but non repairable components. Let $G$ denote the distribution function of the failure time, and $g$ its density. In order to apply Theorem 1.1, one can assume that this distribution is phasetype. The degradation function is the probability of failure at time $t$, i.e. $m(t)=G(t)$. It is strictly increasing, from 0 to 1 . For each $\alpha$ such that $0<\alpha<1$, the mean hitting time $t_{\alpha}$ is the $\alpha$-th quantile of the failure time: $t_{\alpha}=G^{-1}(\alpha)$. The asymptotic variance is now:

$$
\sigma_{\alpha}^{2}=\frac{\alpha(1-\alpha)}{g\left(G^{-1}(\alpha)\right)^{2}}
$$

But if the components are not repairable, the time at which the $k$-th of them fails is the $k$-th order statistic of a sample of i.i.d. failure times, with distribution function $G$. So in this particular case, our result amounts to the well known central limit theorem for order statistics (e.g. Reiss [16], Sect. 4.1, pp. 108-109).

\subsection{Markovian multi-state components}

Our results also apply to multi-state components. The different states of one component correspond to performance levels and describe its progressive degradation. As an example, we will consider an extension of a model studied by Pham et al. in [14] where the cardinality of the state space is 3 . The three states are 


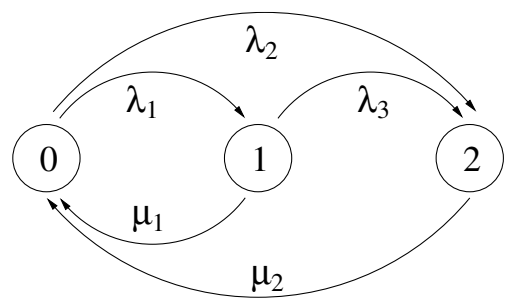

Figure 2. Transition diagram of a three-state component.

0 (perfect), 1 (deteriorated) and 2 (failed). A perfect component can either deteriorate or directly fail. A deteriorated component can either fail or be repaired. A failed component is restored to perfect state. The transition diagram is that of Figure 2. The infinitesimal generator is the following

$$
\Lambda=\left(\begin{array}{ccc}
-\left(\lambda_{1}+\lambda_{2}\right) & \lambda_{1} & \lambda_{2} \\
\mu_{1} & -\left(\mu_{1}+\lambda_{3}\right) & \lambda_{3} \\
\mu_{2} & 0 & -\mu_{2}
\end{array}\right)
$$

The matrix $\exp (\Lambda t)$ can be explicitly computed, but the expressions are somewhat lengthy. Simpler formulas are obtained for $\lambda_{1}=\lambda_{2}=\lambda_{3}=\lambda$. For the probabilities of the different states starting from 0 at time $t=0$, one gets:

$$
\left\{\begin{array}{l}
p_{0}(t)=\frac{\mu_{2}\left(\lambda+\mu_{1}\right)}{\left(2 \lambda+\mu_{1}\right)\left(\lambda+\mu_{2}\right)}+\frac{\lambda\left(2 \lambda+\mu_{1}-\mu_{2}\right)}{\left(2 \lambda+\mu_{1}\right)\left(\lambda+\mu_{1}-\mu_{2}\right)} \mathrm{e}^{-\left(2 \lambda+\mu_{1}\right) t}+\frac{\lambda\left(\mu_{1}-\mu_{2}\right)}{\left(\lambda+\mu_{2}\right)\left(\lambda+\mu_{1}-\mu_{2}\right)} \mathrm{e}^{-\left(\lambda+\mu_{2}\right) t} \\
p_{1}(t)=\frac{\lambda\left(2 \lambda+\mu_{1}-\mu_{2}\right)}{\left(2 \lambda+\mu_{1}\right)\left(\lambda+\mu_{2}\right)}-\frac{\lambda^{2}}{\left(2 \lambda+\mu_{1}\right)\left(\lambda+\mu_{1}-\mu_{2}\right)} \mathrm{e}^{-\left(2 \lambda+\mu_{1}\right) t}+\frac{\lambda}{\left(\lambda+\mu_{2}\right)\left(\lambda+\mu_{1}-\mu_{2}\right)} \mathrm{e}^{-\left(\lambda+\mu_{2}\right) t} \\
p_{2}(t)=\frac{\lambda}{\lambda+\mu_{2}}-\frac{\lambda}{\lambda+\mu_{2}} \mathrm{e}^{-\left(\lambda+\mu_{2}\right) t}
\end{array}\right.
$$

In Pham et al. [14], a failed component is not repaired $\left(\mu_{2}=0\right)$, so state 2 is absorbing. Their system is supposed to be failed as soon as $k$ out of $n$ components are in state 2 . With our notations, this corresponds to $f(0)=f(1)=0$, and $f(2)=1$. The mean degradation is $m(t)=p_{2}(t)$. The mean hitting time $t_{\alpha}$ and the asymptotic variance $\sigma_{\alpha}^{2}$ can also be explicitly computed. The expressions are similar to those of the binary case. For $\alpha<\frac{\lambda}{\lambda+\mu_{2}}$ :

$$
t_{\alpha}=-\frac{1}{\lambda+\mu_{2}} \log \left(1-\frac{\alpha\left(\lambda+\mu_{2}\right)}{\lambda}\right),
$$

and:

$$
\sigma_{\alpha}^{2}=\frac{\alpha(1-\alpha)}{\left(\lambda-\alpha\left(\lambda+\mu_{2}\right)\right)^{2}}
$$

Consider now a variant of this model which takes into account the difference between a perfect and a degraded component. We can choose the function $f$ as follows: $f(0)=0, f(1)=\frac{1}{2}$, and $f(2)=1$. The mean degradation becomes $m(t)=\frac{1}{2} p_{1}(t)+p_{2}(t)$ which may no longer be increasing for all $t$, depending on the value of $\mu_{2}$. For suitable values of $\alpha, t_{\alpha}$ can be computed at least numerically, and Theorem 1.1 can be applied.

Acknowledgements. We are pleased to thank J. Jacod and J. Rousseau for helpful hints. We are deeply indebted to the anonymous referee for his important participation. 


\section{REFERENCES}

[1] S. Asmussen, Applied probability and queues. Wiley, New York (1987).

[2] S. Asmussen, Matrix-analytic models and their analysis. Scand. J. Statist. 27 (2000) 193-226.

[3] T. Aven and U. Jensen, Stochastic models in reliability. Springer, New York (1999).

[4] R.E. Barlow and F. Proschan, Mathematical theory of reliability. SIAM, Philadelphia (1996).

[5] U.N. Bhat, Elements of applied stochastic processes. Wiley, New York (1984).

[6] D. Chauveau and J. Diébolt, An automated stopping rule for MCMC convergence assessment. Comput. Statist. 14 (1999) 419-442.

[7] C. Cocozza-Thivent, Processus stochatisques et fiablité des systèmes. Springer, Paris (1997).

[8] R.M. Dudley, Real analysis and probability. Chapman and Hall, London (1989).

[9] S.N. Ethier and T.G. Kurtz, Markov processes: characterization and convergence. Wiley, New York (1986).

[10] M.G. Hahn, Central limit theorem in D[0,1]. Z. Wahrsch. Verw. Geb 44 (1978) 89-101.

[11] A. Hølyand and M. Rausand, System reliability theory: models and statistical methods. Wiley, New York (1994).

[12] M.F. Neuts, Structured stochastic matrices of $M / G / 1$ type and their applications. Dekker, New York (1989).

[13] M.F. Neuts, Matrix-geometric solutions in stochastic models: an algorithmic approach. Dover, New York (1994).

[14] H. Pham, A. Suprasad and R.B. Misra, Reliability analysis of $k$-out-of- $n$ systems with partially repairable multi-state components. Microelectron. Reliab. 36 (1996) 1407-1415.

[15] D. Pollard, Convergence of stochastic processes. Springer, New York (1984).

[16] R.-D. Reiss, Approximate distributions of order statistics, with application to non-parametric statistics. Springer, New York (1989).

[17] H.C. Tijms, Stochastic models: an algorithmic approach. Wiley, Chichester (1994).

[18] W. Whitt, Some useful functions for functional limit theorems. Math. Oper. Res. 5 (1980) 67-85.

[19] B. Ycart, Cutoff for samples of Markov chains. ESAIM: PS 3 (1999) 89-107.

[20] B. Ycart, Stopping tests for Monte-Carlo Markov chain methods. Meth. Comp. Appl. Probab. 2 (2000) 23-36.

[21] B. Ycart, Cutoff for Markov chains: some examples and applications. in Complex Systems, E. Goles and S. Martínez Eds., Kluwer, Dordrecht (2001) 261-300. 